\title{
IMPROVED COLOR BARCODES VIA EXPECTATION MAXIMIZATION STYLE INTERFERENCE CANCELLATION
}

\author{
Orhan Bulan and Gaurav Sharma \\ ECE Dept., University of Rochester, Rochester, NY, 14627-0126 \\ \{bulan,gsharma\}@ece.rochester.edu
}

\begin{abstract}
Encoding data independently in cyan, magenta, and yellow (CMY) print colorant channels with detection in complementary Red, green, and blue (RGB) image capture channels offers an attractive framework for extending monochrome barcodes to color with increased data rates. The undesired absorption of colorants in regions of spectral sensitivity of the noncomplementary capture channels, however, gives rise to cross-channel color interference that significantly deteriorates the performance of the color barcode system. In this paper, we propose an Expectation Maximization (EM) style algorithm to estimate and cancel this color interference and improve the overall performance of the barcode system. Our method utilizes a physical model for print-capture process where the model parameters vary depending on printer, capture device, and illumination. We estimate the model parameters using an iterative EM-style approach and obtain an estimate of CMY colorant channels from the scanned RGB barcode by using the estimated model parameters. Our experimental results show that the proposed method mitigates the effect of color interference and significantly reduces the bit error rates for the recovered data.
\end{abstract}

Index Terms - high capacity, color barcodes, color interference cancellation, expectation maximization

\section{INTRODUCTION}

Two-dimensional barcodes are commonly used to connect the tangible analog and physical world with digital and networked applications. These applications include tracking trade items for inventory control and billing, encoding biometrics for secure and reliable person identification [1], and improving document quality in scan and print applications [2]. Because barcodes consume precious spatial "real-estate" on physical media, high capacity barcodes that increase the information density per unit area are highly desirable. Recently the latitude offered by color reproduction has been actively studied to increase the capacity of monochrome barcodes [3-9].

Color printing uses cyan $(\mathrm{C})$, magenta $(\mathrm{M})$, and yellow $(\mathrm{Y})$ colorants as the subtractive primaries [10] for color reproduction in printing. Color capture devices use red $(\mathrm{R})$, green $(\mathrm{G})$, and blue (B) sensing channels that are complementary to the CMY colorants. The cyan colorant absorbs primarily in the region over which the red channel of common color capture devices is sensitive; $M$ and $\mathrm{Y}$ colorants have as similar complementary relation with the $\mathrm{R}$ and $\mathrm{B}$ channels, respectively. An attractive option for extending

This work was supported in part by the Xerox Foundation grant, and by a matching grant from New York State Office of Science, Technology and Academic Research (NYSTAR) through the Center for Emerging and Innovative Sciences (CEIS). monochrome barcodes to color is to therefore exploit this complementarity and encode data independently in each of the $\mathrm{C}, \mathrm{M}$, and $\mathrm{Y}$ print channels with a monochrome barcode technique and to recover the data from the complementary R, G, and B channels, respectively. The method is particularly attractive because most image capture devices already provide RGB color capture and because the monochrome barcode techniques are quite mature in their development. The method proposed in [9] offers an example of a barcode using this methodology, which we will use as a specific focused example for our presentation, even though the method we propose here can be more broadly applied.

For the method in [9], the data in each colorant channel is encoded in elliptical-dot arrays where the orientation of individual elliptical dots is modulated for the purpose of encoding data. The data encoded CMY colorant layers are printed in overlay to generate the color barcode. At the decoder, data is recovered from an RGB scan, using the channel complementary to the colorant to recover the data in each colorant layer. As noted previously, the inter-channel color interference poses a challenge in this setting. In [9] this is circumvented by restricting to binary modulation with a carefully designed signaling constellation that mitigates the interference and by using a sequential error correction decoding that comprehends the interference at the decoder.

In this paper, we propose an expectation maximization (EM) style methodology to estimate and cancel color interference from noncomplementary print channels in an RGB capture of a per channel CMY barcode. For this purpose, we utilize a physical model for printing and scanning processes whose parameters vary depending on printer, capture device and illumination etc. In this setting, the model parameters and the CMY print colorants form the hidden and latent variables. We estimate the hidden model parameters using an EM-type iterative approach where a cost function, associated with the physical model, is minimized at each iteration of the algorithm. The CMY colorant channels are then calculated from the RGB scan of the barcode using the estimated model parameters, paving the way for improved decoding of data in each of the channels. Experiments demonstrate that the proposed method mitigates the effect of color interference seen in the scan channels and significantly improve the performance of the overall barcode system. As compared with the method of [9], the framework proposed here offers greater generality, being robust to misregistration errors, applicable to non-binary orientation modulation and also to other per-channel encoding extensions of monochrome barcodes [11].

In Section 2 we summarize the color barcode technology proposed in [9] via per-colorant data encoding and introduce the color interference challenge in this encoding scenario. We describe the proposed methodology to address the color interference challenge to obtain a better estimate of CMY channels in Section 3. Experimental results from tests performed with the proposed interference 
cancellation method are presented in Section 4. Section 5 concludes the paper with a summary of the major results and a discussion.

\section{PER-COLORANT DATA ENCODING VIA ORIENTATION MODULATION}

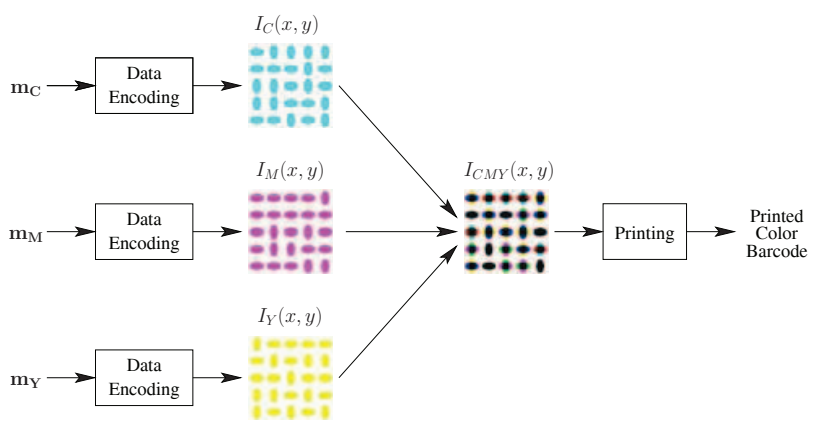

Fig. 1. Per-colorant data encoding via orientation modulation in elliptical-dot arrays.

Fig. 1 shows a block diagram of the per-colorant orientation modulation based data encoding methodology recently proposed in [9]. The message data $\mathbf{m}_{\mathbf{C}}, \mathbf{m}_{\mathbf{M}}$, and $\mathbf{m}_{\mathbf{Y}}$ corresponding to $\mathrm{C}, \mathrm{M}$, and $\mathrm{Y}$ colorant channels, respectively, are encoded in each colorant layer. In order to enable global and local synchronization, a two-dimensional rectilinear tiling is defined across the barcode substrate, where in each cell resulting from the tiling an elliptical dot is placed at the center. The orientation of the elliptical-dot in each cell is modulated to carry the message data. The same tiling is defined for all colorant layers and the corresponding binary images $I_{C}(x, y), I_{M}(x, y)$, and $I_{Y}(x, y)$ are generated. The resulting binary images are printed in overlay to generate the barcode.

As illustrated in Fig. 2, embedded data in each colorant channel is extracted from the complementary capture channel in a conventional RGB scan of the barcode. Specifically, the rectilinear array allows for estimation of rotation and scaling providing global synchronization; after which, the orientation within each cell is estimated by computing image moments along each of the embedding orientations, with the computed moments also providing local synchronization to overcome geometric distortion in the printing process [9]. A fundamental challenge for data extraction is the interchannel color interference, also seen in the individual channel images shown in Fig. 2. The RGB scan channels not only capture the information from the complementary print channel but also see interference from the other print colorants. This interference is most obvious for the blue (B) channel, which captures not only the pattern printed with the yellow colorant but also sees significant interference from the magenta colorant.

As opposed to other high capacity barcode technologies [3-6], the method encodes data in the shape of elliptical-dots in colorant channels instead of the color or graylevel of a cell. Embedding in orientation provides robustness against the variations in printing and scanning systems, which preserve shapes and therefore orientation (within resolution limits) but are subject to tonal variations in gray levels across devices and over time, which can adversely impact the detection accuracy for encoding in color gray values [3-6]. Dataglyphs $[7,8]$ share similar motivation but in their color instantiation use the same orientations for the different channels and therefore offer no data rate improvements with color. They also differ in that

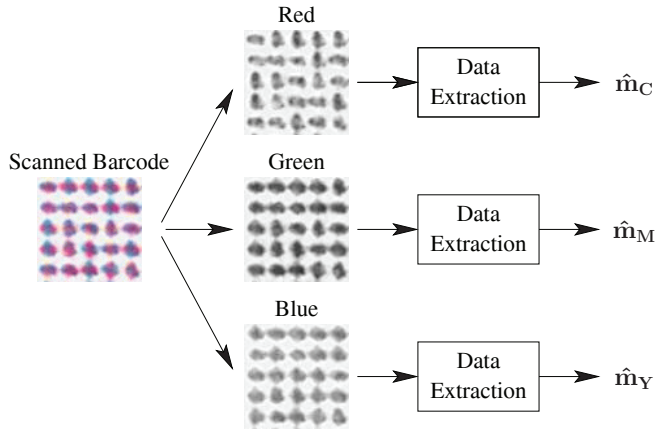

Fig. 2. Data extraction from RGB scan channels.

they use correlation instead of image moments for detection, requiring finer alignment/synchronization.

\section{EXPECTATION MAXIMIZATION STYLE COLOR INTERFERENCE CANCELLATION}

To overcome the cross-channel color-interference, we utilize a physical model for the print and capture process. Assuming the C, M, and Y colorants are transparent (non-scattering), we model the print using the the transparent version of Kubelka-Munk theory or the BeerBouguer law [12, Chap. 7] [10], as $\left(t_{i}(\lambda)\right)^{2}=10^{-d_{i}(\lambda)}$, where $d_{i}(\lambda)$ is the optical density for the $i^{t h}$ colorant layer (under to and fro passage), where $(i \in\{C, M, Y\})$. The binary colorant layers of the barcode are represented as bi-level images $I_{i}(x, y)(i \in$ $\{C, M, Y\})$, where $I_{i}(x, y)$ is ' 1 ' in regions where the $i^{t h}$ colorant is printed and ' 0 ', elsewhere. The spatial pattern of spectral reflectance of the printed barcode can then be approximated as [10]:

$$
r(x, y ; \lambda)=r_{w}(\lambda) 10^{-\sum_{i \in\{C, M, Y\}} d^{i}(\lambda) I_{i}(x, y)}
$$

where $r_{w}(\lambda)$ is the spectral reflectance of the barcode substrate (paper).

If the spectral sensitivity of the R, G, B capture channels is represented by $s_{k}(\lambda), k \in\{R, G, B\}$, the three channels in the image captured by the digital camera can be expressed as $I_{k}^{s}(x, y)=$ $\int s_{k}(\lambda) r(x, y ; \lambda) d \lambda, k \in\{R, G, B\}$. We further approximate the spectral sensitivities of R, G, B camera color channels as Dirac delta functions $s_{k}(\lambda)=\alpha_{k} \delta\left(\lambda-\lambda_{k}\right)$ at corresponding wavelengths $\lambda_{k}$, $k \in\{R, G, B\}$, which allows us to write the normalized optical densities corresponding to the three captured channels as

$$
d_{k}(x, y) \stackrel{\text { def }}{=}-\log _{10}\left(\frac{I_{k}^{s}(x, y)}{I_{k}^{s}(W)}\right)=\sum_{i \in\{C, M, Y\}} d_{k}^{i} I_{i}(x, y)
$$

where $I_{k}^{s}(W)$ is the captured image value corresponding to the paper substrate in the $k^{t h}$ camera channel and $d_{k}^{i}=d^{i}\left(\lambda_{k}\right)$.

The linear relation in (2) between the densities corresponding to camera responses and print colorant channels allows us to perform interference cancellation and estimate the print colorant channels from the camera color channels provided that the optical density of the colorant layers in the camera channels $d_{k}^{i}$ 's for all $k \in$ $\{R, G, B\}$ and $i \in\{C, M, Y\}$ are available. Specifically, denoting by $\mathbf{D}$ the matrix whose $i^{\text {th }}$ column is $\left[d_{R}^{i}, d_{G}^{i}, d_{B}^{i}\right]^{T}$, we see that under the model of (2) the printed binary channels $\mathbf{I}=\left[I_{C}, I_{M}, I_{Y}\right]^{T}$ at a given location $(x, y)$ are obtained from the recorded density 
$\mathbf{d}=\left[d_{R}^{i}, d_{G}^{i}, d_{B}^{i}\right]^{T}$ at that location by

$$
\mathbf{I}=\mathbf{D}^{-1} \mathbf{d}
$$

Based on (3), our goal is to estimate the printed binary channels I from the density matrix $\mathbf{d}$ calculated from the captured barcode. In a practical applications, however, we typically do not know $\mathbf{D}$, which varies, depending, among other factors, on the printer colorants, capture device, and illumination. We therefore use an iterative algorithm to estimate $\mathbf{I}$ and $\mathbf{D}$, treating these quantities as hidden and latent variables in an expectation maximization (EM) style formulation. Specifically, we relax the integer constraints on $\mathbf{I}$, treating these instead as variables constrained to lie between 0 and 1 , which may alternatively be interpreted as probabilities of the corresponding colorant being printed at the given pixel location. We then formulate the problem of estimating $\mathbf{I}$ and $\mathbf{D}$ as an optimization problem that seeks to minimize the cost function $\|\mathbf{D I}-\mathbf{d}\|^{2}$. Because we have hidden and latent variables in the cost function, we formulate the overall estimation process as an alternating least squares minimization as indicated in Algorithm 1. Each iteration of the algorithm alternates over minimization of the cost function over $\mathbf{D}$ and $\mathbf{I}$ in two sequential steps. In this process, the constraint $0 \leq \mathbf{I} \leq 1$ is imposed based on our proposed relaxation and additionally, based on the physical model, we constrain $\mathbf{D}$ to include only positive entries. The algorithm is initialized by setting $\mathbf{D}$ to $3 \times 3$ identity matrix ${ }^{1}$. The algorithm is terminated once the improvement in the objective function falls below a predetermined threshold $\tau$. Note that the relaxation of the integer constraint on $\mathbf{I}$ in Algorithm 1 also renders the problem computationally tractable; the integer constrained problem is NP hard [13]. For computational and memory efficiency, the computations for Algorithm 1 may be performed over a subset of the pixels in the image. The print colorant channels I can then be estimated by using (3) with the estimate of $\mathbf{D}$ obtained via Algorithm 1.

Note that convergence of the alternating minimization algorithm is assured because each step in the iteration reduces the value of the cost function. In view of the probabilistic interpretation of the relaxation we introduced, the proposed methodology to find $\mathbf{D}$ is an EM-type algorithm that finds the local optima in the vicinity of the initialization point [14, Chap. 9].

\section{EXPERIMENTAL RESULTS}

The performance of the proposed color interference cancellation algorithm was evaluated experimentally using the orientation modulation color barcode scheme outlined in Section 2. A $2 \times 2$ inch color barcode was generated and printed on a HP Color 4700 printer with an array periodicity of 75 cells per inch (cpi) along horizontal and vertical directions for each of the CMY channels. This yields cell sizes (in printer pixel units) that provide reasonable flexibility to orient a dot along different directions within a cell and an overall data density of 16,875 bits per square inch. In each colorant channel we modulate the dot orientation along vertical or horizontal directions (i.e. $0 / 90^{\circ}$ orientations) based on the randomly generated data to be encoded in the barcode. The barcode was scanned on an HP Scanjet 8270 scanner with a scanning resolution of a 1200 dots per inch (dpi) resolution for data extraction ${ }^{2}$. Fig. 3 illustrates a portion of the scanned barcode and the color channels of the barcode be-

\footnotetext{
${ }^{1}$ This corresponds to the idealized assumption that the RGB sensor channels capture the printing in the complementary CMY colorants with no color interference.

${ }^{2}$ Preliminary tests have also been conducted on other printer and scanner combinations with results similar to our chosen combination.
}

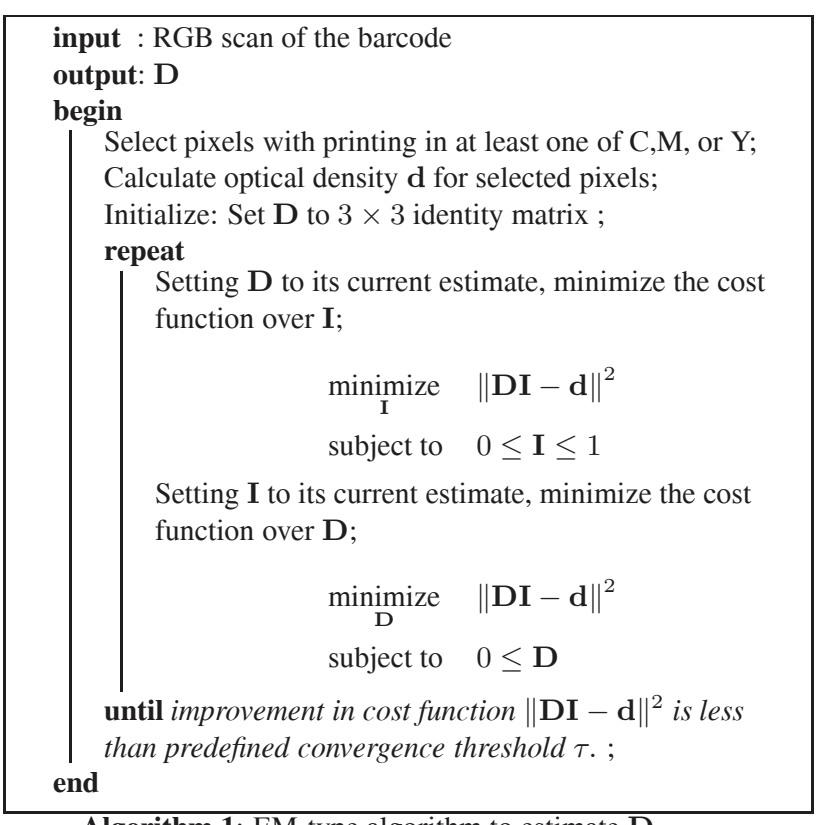

Algorithm 1: EM-type algorithm to estimate D.

fore and after color interference cancellation algorithm (a value of $\tau=10^{-5}$ was used for the convergence check). Note that the estimated cyan, magenta, and yellow channels with the proposed algorithm for color interference cancellation exhibit reduced color interference compared with the original RGB channels. The interference and the reduction are more pronounced when the dot orientations in the neighboring colorant channels in the spectra (i.e. cyan-magenta or magenta-yellow), do not match with each other.

Next we evaluate the bit error rates (BER) for the data embedded in each of the colorant channels in the barcodes, comparing the performance with and without color interference cancellation. The data is extracted from the estimated print colorant channels. When the proposed color interference cancellation is not employed, the data is extracted directly from RGB channels of the scanned barcode complementary to CMY print colorant channels, respectively. In each color channel, we employ Otsu's thresholding algorithm [15] to mitigate the effect of color interference and reduce the print-scan noise that impacts the estimated orientation of elliptical-dots. After global and local synchronization, the data is extracted from the thresholded color channels by utilizing image moments as detection statistics as outlined in Section 2 and detailed in [9]. Table 1 lists the BER performance in estimated CMY colorant channels with and without color interference cancellation. Note that the proposed algorithm for interference cancellation significantly improves the BER performance especially in magenta and yellow channels, which are typically impacted by the color interference more dominantly [9]. Also note that the BER performance in the cyan channel is not improved by the proposed algorithm as the red scan channel captures the cyan colorant with minimal interference from the other colorants [9], which is typically eliminated with the adaptive thresholding.

The convergence behavior of the algorithm is illustrated in Fig. 4, where the graph shows a plot of the optimization objective function as a function of the iteration number. Consistent with the remarks of Section 3, monotone convergence to a (local) minimum (in the vicinity of the initialization) is observed. 


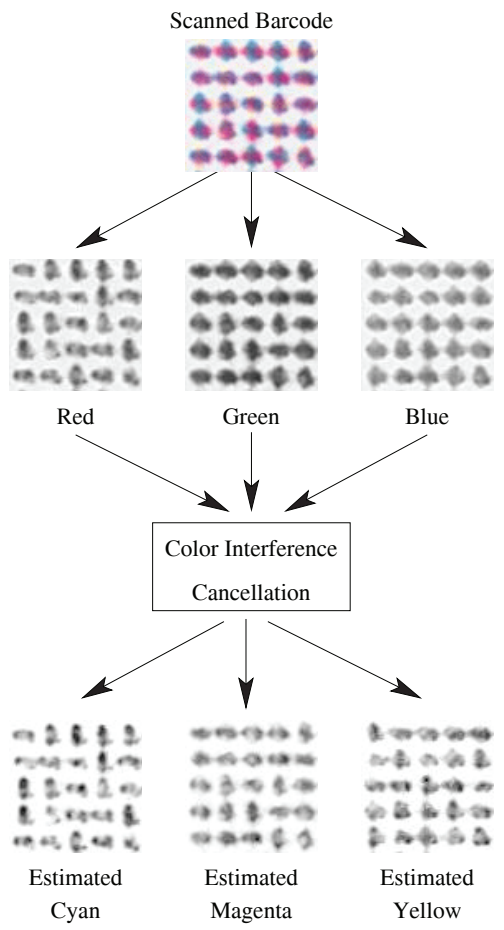

Fig. 3. Scanned RGB channels and estimated CMY channels after interference cancellation.

\begin{tabular}{|c|c|c|c|}
\cline { 2 - 4 } \multicolumn{1}{c|}{} & $\begin{array}{c}\text { Estimated } \\
\text { Cyan }\end{array}$ & $\begin{array}{c}\text { Estimated } \\
\text { Magenta }\end{array}$ & $\begin{array}{c}\text { Estimated } \\
\text { Yellow }\end{array}$ \\
\hline $\begin{array}{c}\text { Without Int. } \\
\text { Cancellation }\end{array}$ & 0.10 & 0.03 & 0.28 \\
\hline $\begin{array}{c}\text { Proposed EM-style } \\
\text { Cancellation }\end{array}$ & 0.10 & 0 & 0.10 \\
\hline
\end{tabular}

Table 1. Bit error rates in color channels, obtained from RGB scan channels and by utilizing the proposed color interference cancellation algorithm.

\section{CONCLUSION AND DISCUSSION}

The EM-style algorithm formulated in this paper provides an effective framework for improving the performance of high capacity color barcodes based on per-colorant channel data encoding by allowing estimation and cancellation of inter-colorant interference. The proposed method significantly improves the bit error rate performance of the barcode system, especially in magenta and yellow channels, where the color interference is the most pronounced due to undesirable coupling between printer colorant and scanner channels.

We also observe that Algorithm 1 provides a method for estimating a three channel image corresponding to $\mathrm{CMY}$ print colorants from a three channel RGB scanned image and is not directly coupled with the data embedding and recovery process outlined in Section 2. Its applicability is therefore not constrained to the specific barcode scheme or to the specific application. As noted previously, our presentations uses a focused example for clear motivation and to clearly quantify the advantage in the chosen application setting.

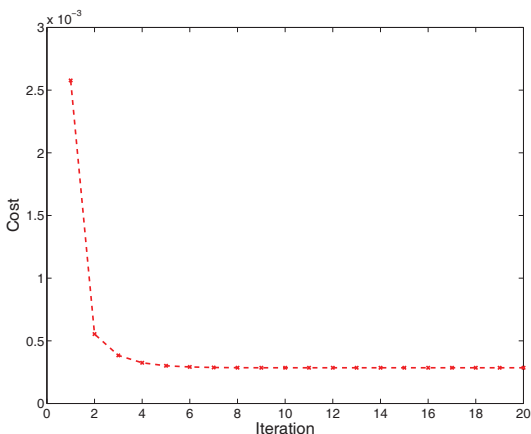

Fig. 4. Objective function vs iteration number illustrating convergence behavior of the proposed estimation algorithm.

\section{REFERENCES}

[1] D. Schonberg and D. Kirovski, "EyeCerts," IEEE Transactions on Information Forensics and Security, vol. 1, no. 2, pp. 144-153, 2006.

[2] S. Voloshynovskiy, O. Koval, F. Deguillaume, and T. Pun, "Visual communications with side information via distributed printing channels: extended multimedia and security perspectives," in Proc. SPIE: Security, Steganography, and Watermarking of Multimedia Contents VI, E. J. Delp and P. W. Wong, Eds., vol. 5306, Jan. 2004, pp. 428-445.

[3] "High capacity color barcodes," accessed Jan 2010. [Online]. Available: http://research.microsoft.com/en-us/projects/hccb/

[4] D. Parikh and G. Jancke, "Localization and Segmentation of A 2D High Capacity Color Barcode," in IEEE Workshop on Applications of Computer Vision (WACV) 2008., 2008, pp. 1-6.

[5] S. J. Simske, J. S. Aronoff, and M. Sturgill, "Revenge of the physical mobile color barcode solutions to security challenges," in Proc. Optical Document Security, San Francisco, 20-22 Jan. 2010, pp. 184-197.

[6] J. Mayer, J. Bermudez, A. Legg, B. Uchoa-Filho, D. Mukherjee, A. Said, R. Samadani, and S. Simske, "Design of high capacity 3D print codes aiming for robustness to the PS channel and external distortions," in Proc. IEEE Intl. Conf. Image Proc., Nov. 2009, pp. 105-108.

[7] D. L. Hecht, "Printed embedded data graphical user interfaces," IEEE Computer, vol. 34, no. 3, pp. 47-55, Mar. 2001.

[8] —, "Embedded data glyph technology for hardcopy digital documents," in Proc. SPIE: Color hard copy and graphic arts III, J. Bares, Ed., vol. 2171, Mar. 2001, pp. 341-352.

[9] O. Bulan and G. Sharma, "High capacity color barcodes: Per channel data encoding via orientation modulation in elliptical dot arrays," IEEE Trans. Image Proc., vol. 20, no. 5, pp. 1337-1350, May 2011. [Online].

[10] G. Sharma and H. J. Trussell, "Digital color imaging," IEEE Trans. Image Proc., vol. 6, no. 7, pp. 901-932, Jul. 1997.

[11] O. Bulan, H. Blasinski, and G. Sharma, "Color QR codes: Increased capacity via per-channel data encoding and interference cancellation," in Proc. IS\&T/SID Nineteenth Color and Imaging Conference: Color Science and Engineering Systems, Technologies, and Applications, San Jose, CA, 7-11 Nov. 2011, accepted for publication.

[12] F. Grum and C. J. Bartleson, Eds., Optical Radiation Measurements: Color Measurement. New York: Academic Press, 1980, vol. 2.

[13] X. Chang and Q. Han, "Solving box-constrained integer least squares problems," IEEE Transactions on Wireless Communications, vol. 7 , no. 1, pp. 277-287, 2008.

[14] C. Bishop, Pattern recognition and machine learning. New York, NY: Springer, 2006

[15] N. Otsu, "A threshold selection method from gray-level histograms," IEEE Trans. Sys., Man, and Cyber., vol. 9, no. 1, pp. 225-236, Jan. 1979. 